\title{
It's Time for a Haircut: a Perspective on Barbershop Health Interventions Serving Black Men
}

\author{
Kelsey B. Bryant, MD, MPH' , C. Adair Blyler, PharmD², and Robert E. Fullilove, EdD ${ }^{3}$ \\ 'Division of General Medicine, Columbia University Irving Medical Center, New York, NY, USA; ${ }^{2}$ Hypertension Center of Excellence, Smidt Heart \\ Institute, Cedars-Sinai Medical Center, Los Angeles, CA, USA; ${ }^{3}$ Mailman School of Public Health, Columbia University Irving Medical Center, New \\ York, NY, USA
}

Black men in the USA experience disproportionate cardiovascular disease mortality compared to their white counterparts, in part due to an excess of uncontrolled hypertension. A promising intervention to address these disparities involves the direct pharmacologic management of hypertension by clinical pharmacists in Black male patrons of barbershops, as demonstrated in the Los Angeles Barbershop Blood Pressure Study (LABBPS). Despite the observed reduction in systolic blood pressure of $>20 \mathrm{mmHg}$ after 1 year, the feasibility of scaling up such an intervention to a regional or national platform remains uncertain. Here we explore the success of LABBPS in the context of prior barbershop interventions and theorize the most important aspects driving the observed reductions. We further make a case for prioritizing preventive care in nontraditional settings in an effort to reduce health disparities.

J Gen Intern Med 35(10):3057-9

DOI: $10.1007 / \mathrm{s} 11606-020-05764-8$

(c) Society of General Internal Medicine 2020

$\mathrm{C}$ onfronting health disparities is an enduring priority in the USA. Hypertension - the leading modifiable risk factor for leading cause of death ${ }^{1}$ - remains one of the most significant challenges for the health of Black Americans. ${ }^{2}$ With higher rates of hypertension diagnoses, they have poorer rates of control than any other racial and ethnic group in the nation. ${ }^{3}$ In turn, they bear a higher burden of cardiovascular disease (CVD) and associated mortality. ${ }^{4-6}$ Low-cost, effective treatment exists, but the traditional method of delivering care has not succeeded in eliminating these disparities in the Black community. ${ }^{7}$

In collaboration with colleagues, Dr. Bryant presented preliminary results of a 10-year simulation of expected reduction in blood pressure and cardiovascular disease events using LABBPS data at SGIM 2019 in Washington DC as well as preliminary results of a 10-year simulation estimating costs of LABBPS implementation at the 2019 American Heart Association Hypertension meeing in New Orleans, LA.

Received February 14, 2020

Accepted February 28, 2020

Published online March 16, 2020
Thankfully, some people have gotten creative.

Led by the late Dr. Ronald Victor, the Los Angeles Barbershop Blood Pressure Study (LABBPS) enrolled a cohort of non-Hispanic Black men with uncontrolled hypertension from Black-owned barbershops in Los Angeles County. A pharmacist-driven intervention involving the direct management of antihypertensive medication in the barbershops themselves was delivered. The results were impressive: a greater than $20 \mathrm{mmHg}$ reduction in systolic blood pressure translating to $64 \%$ with blood pressure $<130 / 80 \mathrm{mmHg}$ at 6 months and sustained at 1 year. ${ }^{8,9}$

Community health promotion programs are hardly unique, ${ }^{10-12}$ nor is the use of barbershops to house such interventions. ${ }^{13-18}$ What is unique, however, is having clinical evidence that engaging community members in chronic disease management outside of the clinic walls can work. ${ }^{8,9}$ While there are many questions regarding the scalability and sustainability of LABBPS, ${ }^{19,} 20$ exploring the underlying reasons for success and drawing from these to design future interventions - whether or not they occur in barbershops-is a worthwhile exercise.

In a perspective piece, Cooper et al. poignantly stated "race captures a lifelong social experience". ${ }^{21}$ Race is understood to be a social construct that encompasses a complex set of factors from generational wealth and economic disadvantage to being at elevated, undeserved risk for incarceration. ${ }^{22}$ These risks and the body's reaction to them have real biological consequences. Untangling the factors that contribute to hypertension among Black men is a complex task, but it is possible that blood pressure control in a nontraditional environment, barbershop or elsewhere, is a step towards health equity.

LABBPS demonstrated that it is possible to lower blood pressure in individuals who had not achieved control in a traditional clinical setting. ${ }^{8,} 9$ In fact, almost $80 \%$ of participants in LABBPS had a primary provider, yet their blood pressure was still uncontrolled. ${ }^{8}$ It is not clear exactly which component of the LABBPS intervention drove success, though qualitative research is underway to better understand the most important aspects. ${ }^{19,20}$ Nonetheless, access to care in a familiar, trusted setting with algorithm-driven management and support from a barber (read: friend) appears to have interacted in a powerful way to achieve heretofore unheard of levels of success: a population of men with baseline blood 
pressures $>150 / 90 \mathrm{mmHg}$ to more the $60 \%$ those achieving the AHA $/ \mathrm{ACC}^{23}$-recommended level of $<130 / 80 \mathrm{mmHg}$.

A 2014 review detailed various barber and beauty shop community health interventions, including more than 50 studies involving health promotion in nonclinical settings. The advantage in such venues is that they have historically been part of the social, economic, and political framework of communities large and small across the country. ${ }^{13}$ These locales are strongly connected to the everyday lives of their clients, and the sense of community is powerful. Well-established barbershops have developed trusting relationships between barbers and customers that have been years in the making. For example, customers in LABBPS cited on average 10 years of patronage with biweekly visits. ${ }^{8}$ This trial appears to have been perceived as an extension of this existing, trusting relationship that helped to produce a set of highly successful clinical outcomes.

The compelling effect of the barbershop is likely multifactorial, but trust leveraged in partnering with the community in a comfortable space is fundamental. Traditional healthcare happens in a locale that feels safe to those employed in the industry. For too many health workers, clinical work has become routine: it is what we were trained to do in the places we were trained to do it. That is not the experience of our patients, and some have a harder time than others finding the trusting connection they need. Though the pharmacists delivering care in LABBPS were not community members, their collaboration with barbers produced powerful social capital that may not be replicable within the walls of a clinic. For the groups of people our system has failed, we must step outside of our comfort zone and meet them where they are if we are to eliminate health inequity.

As technologic advancements accelerate, medical discoveries are not left behind. With the promise of using the patient's unique genetic make-up to match them to care, precision medicine charges forward as a very costly, but promising, future of medicine. While these advances are nothing short of incredible, Black men, who represent just one group with persistently poor outcomes compared to others, are still dying disproportionately from CVD. ${ }^{2}$ The tragedy is that hypertension and CVD prevention is one area well versed in managing, yet we still struggle to achieve widespread control in marginalized communities.

In 2016, Khoury et al. described precision public health as "the right intervention for the right population at the right time". 24 Perhaps LABBPS is an example of this. However, barriers to dissemination must be addressed, ${ }^{19}, 20$ the most important of which is cost. Secondary economic analyses are underway to address some of these questions. If cost-effectiveness in the context of reduced CVD events and improved quality of life is demonstrated, perhaps a program can be developed that would enable many to enjoy a longer, more functional life. None of this, however, takes into account the social value of reducing health disparities, which one can argue is invaluable. Imagine a world where a population with historically subpar blood pressure control and associated excess risk of $\mathrm{CVD}^{2}$ has a substantially reduced risk as a result of success with a single outcome target: blood pressure control. If we are looking for a focus to reduce cardiovascular morbidity and mortality, why not sever one of the biggest roots of the tree: hypertension?

Of course it is impractical, if not impossible, to create a mini-hypertension clinic in every barbershop across the country. Furthermore, barbershops are among many community locations with potential to create a dramatic reduction in some health disparity. The key is in recognizing that we need to take a different approach to certain aspects in the delivery of preventive care. Ultimately, it is less about physical location and more about the carefully designed efforts to achieve hypertension control in high-risk population that historically has not enjoyed the same health benefits as their white counterparts. Collaboration between respected community members and healthcare entities is one such design that may be especially effective in individuals with uncontrolled hypertension who have not achieved control in traditional clinical settings or lack access to such care altogether. ${ }^{22}$

For an intervention such as LABBPS to become feasible, we must harness any framework that already exists and build upon it, beginning with areas of most need. For example, a provision of the Affordable Care Act (ACA) mandates nonprofit hospitals to conduct community health needs assessments in their surrounding areas to identify issues of highest public health concerns. This practice provides the basis for identifying at-risk populations, and, because the assessments are to occur every 3 years, provides a mechanism for followup. The ACA also mandates community approvals for both the scope and the objectives of such assessments before and after they are implemented. Such community approval must be given at the point when findings are to be published and when recommendations for appropriate interventions are made that are based on those findings. Using this process as a way of engaging and strengthening community partnerships has substantial potential. This of course will not apply to regions served by for-profit institutions, or communities without reliable access to care in any form. It is, however, a possible avenue to engage some at-risk communities, allowing us to reduce disparities when and where we can on a path to eliminating them altogether.

Any effective healthcare initiative takes time and money. When resources are limited, they must be used with maximum efficiency for sustainability. Being conservative with healthcare finances should not deter the development of programs such as barbershop-based hypertension initiatives that have so much potential to reach populations with excess risk. A balance can be struck in the allocation of resources to support cutting-edge developments while providing quality preventive care to all. As healthcare providers and public health professionals, we must orchestrate a coordinated effort utilizing existing resources to create an efficient hypertension management plan on a larger scale than LABBPS - whether or not the intervention occurs in a barbershop. Finding 
individuals will not be hard; the people that stand to benefit most are out there, and they are waiting in line to have their hair cut.

Corresponding Author: Kelsey B. Bryant, MD, MPH; Division of General Medicine Columbia University Irving Medical Center, New York, NY, USA (e-mail: kb2828@cumc.columbia.edu).

Funding Information Dr. Bryant is supported by training grant T32HP10260 (PD: Shea).

\section{Compliance with Ethical Standards:}

Conflict of Interest: The authors declare that they do not have a conflict of interest.

\section{REFERENCES}

1. Fryar CD, Ostchega Y, Hales CM, Zhang G, Kruszon-Moran D. Hypertension Prevalence and Control Among Adults: United States, 2015-2016. NCHS Data Brief. 2017;(289):1-8.

2. Clark D, 3rd, Colantonio LD, Min YI, et al. Population-Attributable Risk for Cardiovascular Disease Associated With Hypertension in Black Adults. JAMA Cardiol. 2019:1-9.

3. Hales CM, Carroll MD, Simon PA, Kuo T, Ogden CL. Hypertension Prevalence, Awareness, Treatment, and Control Among Adults Aged >/=18 Years - Los Angeles County, 1999-2006 and 2007-2014. MMWR Morb Mortal Wkly Rep. 2017;66(32):846-849.

4. Thomas AJ, Eberly LE, Davey Smith G, Neaton JD, Stamler J. Race/ethnicity, income, major risk factors, and cardiovascular disease mortality. Am J Public Health. 2005;95(8): 1417-1423.

5. Jolly S, Vittinghoff E, Chattopadhyay A, Bibbins-Domingo $\mathbf{K}$. Higher cardiovascular disease prevalence and mortality among younger blacks compared to whites. Am J Med. 2010;123(9):811-818.

6. Rodriguez JM, Geronimus AT, Bound J, Dorling D. Black lives matter: Differential mortality and the racial composition of the U.S. electorate, 1970-2004. Soc Sci Med. 2015;136-137:193-199.

7. Mueller M, Purnell TS, Mensah GA, Cooper LA. Reducing racial and ethnic disparities in hypertension prevention and control: what will it take to translate research into practice and policy? Am J Hypertens. 2015;28(6):699-716.

8. Victor RG, Lynch $\mathbf{K}$, Li N N, et al. A Cluster-Randomized Trial of BloodPressure Reduction in Black Barbershops. N Engl J Med. 2018;378(14):1291-1301.

9. Victor RG, Blyler CA, Li N, et al. Sustainability of Blood Pressure Reduction in Black Barbershops. Circulation. 2019;139(1):10-19.
10. Schoenthaler AM, Lancaster KJ, Chaplin W, Butler M, Forsyth J, Ogedegbe G. Cluster Randomized Clinical Trial of FAITH (Faith-Based Approaches in the Treatment of Hypertension) in Blacks. Circ Cardiovasc Gual Outcomes. 2018;11(10):e004691.

11. Lawlor ER, Bradley DT, Cupples ME, Tully MA. The effect of community-based interventions for cardiovascular disease secondary prevention on behavioural risk factors. Prev Med. 2018;114:24-38.

12. Ifeanyi Chiazor E, Evans M, van Woerden H, Oparah AC. A Systematic Review of Community Pharmacists' Interventions in Reducing Major Risk Factors for Cardiovascular Disease. Value Health Reg Issues. 2015;7:9-21.

13. Linnan LA, D'Angelo H, Harrington CB. A literature synthesis of health promotion research in salons and barbershops. Am $J$ Prev Med. 2014;47(1):77-85.

14. Releford BJ, Frencher SK, Jr., Yancey AK. Health promotion in barbershops: balancing outreach and research in African American communities. Ethn Dis. 2010;20(2):185-188.

15. Rader F, Elashoff RM, Niknezhad S, Victor RG. Differential treatment of hypertension by primary care providers and hypertension specialists in a barber-based intervention trial to control hypertension in Black men. Am J Cardiol. 2013;112(9): 1421-1426.

16. Hess PL, Reingold JS, Jones J, et al. Barbershops as hypertension detection, referral, and follow-up centers for black men. Hypertension. 2007;49(5): 1040-1046.

17. Releford BJ, Frencher SK, Jr., Yancey AK, Norris K $\mathbf{K}$. Cardiovascular disease control through barbershops: design of a nationwide outreach program. J Natl Med Assoc. 2010;102(4):336-345.

18. Osorio M, Ravenell JE, Sevick MA, et al. Community-Based Hemoglobin AlC Testing in Barbershops to Identify Black Men With Undiagnosed Diabetes. JAMA Intern Med. 2020.

19. Margolis KL. Inventing a New Model of Hypertension Care for Black Men. N Engl J Med. 2018;378(14):1345-1347.

20. Ferdinand KC, Graham RM. Uncontrolled Hypertension in Black Men. Circulation. 2019;139(1):20-23.

21. Cooper RS, Nadkarni GN, Ogedegbe G. Race, Ancestry, and Reporting in Medical Journals. Jama. 2018;320(15):1531-1532.

22. Bartolome RE, Chen A, Handler J, Platt ST, Gould B. Population Care Management and Team-Based Approach to Reduce Racial Disparities among African Americans/Blacks with Hypertension. Perm J. 2016;20(1):53-59.

23. Whelton PK, Carey RM, Aronow wS, et al. 2017 ACC/AHA/AAPA/ABC/ ACPM/AGS/APhA/ASH/ASPC/NMA/PCNA Guideline for the Prevention, Detection, Evaluation, and Management of High Blood Pressure in Adults: A Report of the American College of Cardiology/American Heart Association Task Force on Clinical Practice Guidelines. Circulation. 2018;138(17):e484-e594.

24. Khoury MJ, Iademarco MF, Riley WT. Precision Public Health for the Era of Precision Medicine. Am J Prev Med. 2016;50(3):398-401.

Publisher's Note: Springer Nature remains neutral with regard to jurisdictional claims in published maps and institutional affiliations. 